\title{
Impactos das Mudanças Climáticas no Bioma Caatinga na Percepção dos Professores da Rede Pública Municipal de General Sampaio - Ceará
}

\author{
Rafaela Gomes de Almeida ${ }^{1}$, Arnóbio de Mendonça Barreto Cavalcante ${ }^{1,2}$, \\ Emerson Mariano da Silva ${ }^{1}$ \\ ${ }^{1}$ Mestrado Profissional em Climatologia e Aplicações nos Países da CPLP e África, \\ Universidade Estadual do Ceará, Fortaleza, CE, Brasil. \\ ${ }^{2}$ Instituto Nacional de Pesquisas Espaciais, Centro Regional do Nordeste, Unidade de Eusébio, \\ Eusébio, CE, Brasil.
}

Recebido em: 9 de Abril de 2020 - Aceito em: 21 de Maio de 2020

\begin{abstract}
Resumo
O bioma Caatinga é o lugar onde vivem 28,6 milhões de pessoas e que tem sido apontado como uma região ecológica vulnerável às mudanças climáticas. Isso abre a possibilidade para adoção de medidas adaptativas na população em um futuro próximo. Nessa perspectiva, a conscientização da população pode ajudar no processo de adaptação às mudanças climáticas, conscientização que pode ser alcançada por meio da educação formal e a partir da percepção dos agentes sociais. Porém, estariam os agentes sociais, especificamente, os professores percebendo as mudanças climáticas? O presente estudo teve como objetivo avaliar o nível de percepção dos professores da rede pública do município de General Sampaio (CE), em relação aos impactos das mudanças climáticas no bioma Caatinga. Para tanto, foi realizada uma pesquisa de campo por meio de entrevistas aplicadas em 10 professores do Ensino Fundamental, que lecionam nas disciplinas de Ciências e Geografia. As entrevistas foram processadas no software IRAMUTEQ, um método informatizado de análises textuais e analisadas de forma qualitativa. Os resultados mostraram que os professores percebem as variações climáticas por meio de sensações térmicas inconstantes, secas extremas e chuvas fora de época. Todavia, não reconhecem os riscos que o bioma Caatinga e a população estão correndo diante da ameaça das mudanças climáticas.
\end{abstract}

Palavras-chave: clima, ensino fundamental, adaptação climática, iramuteq.

\section{Impacts of Climate Change on the Caatinga Biome in the Perception of Teachers from the Municipal Public Network of General Sampaio - Ceará}

\begin{abstract}
The Caatinga biome is the place where 28.6 million people live and has been identified as an ecological region vulnerable to climate change. This opens the possibility for the adoption of adaptive measures in the population in the near future. In this perspective, the population's awareness can help in the process of adapting to climate change, which can be achieved through formal education and from the perception of social agents. However, are social agents, specifically, elementary school teachers perceiving climate change? The present study sought to analyze the perception level of public school teachers in the municipality of General Sampaio (CE), in relation to the impacts of climate change on the Caatinga biome. To this end, a field research was carried out with interviews involving 10 elementary school teachers, who teach in the disciplines of Science and Geography. The interviews were processed using the IRAMUTEQ software, a computerized method of textual analysis and analyzed in a qualitative way. The results showed that teachers perceive climatic variations, through changing thermal sensations, extreme droughts and off-season rain. However, they do not recognize the risks that the Caatinga biome and the population are taking in the face of the threat of climate change.
\end{abstract}

Keywords: climate, elementary school, climatic adaptation, iramuteq.

Autor de correspondência: Arnóbio de M.B. Cavalcante, arnobio.cavalcante@inpe.br. 


\section{Introdução}

A questão das mudanças climáticas alcançou, recentemente, o topo das agendas científica e política mundiais, um reconhecimento aos impactos negativos sobre a qualidade de vida humana, bem como sobre a vida como um todo no planeta. As evidências que sustentam a ocorrência desse fenômeno estão referenciadas no Quinto Relatório de Avaliação das Mudanças Climáticas (AR5) elaborado pelo Painel Intergovernamental sobre as Mudanças Climáticas - IPCC, que reafirmou as alterações climáticas com citações de impactos em todos os continentes e oceanos (IPCC, 2013; 2014 a,b).

As mudanças climáticas referem-se às alterações nas características do clima da Terra, alterações que podem ser identificadas cientificamente por meio de testes estatísticos e modelagem, levando-se em conta um longo período de tempo. Tais mudanças climáticas estão acontecendo e a relação com as atividades humanas são inequívocas, de modo que, as mudanças climáticas são um dos maiores desafios da humanidade para esse século (IPCC, 2013; 2014a,b).

O IPCC (2013) apontou que a concentração dos gases de efeito estufa (GEE) na atmosfera, tem aumentado expressivamente em comparação aos níveis pré-industriais, assim, sendo considerada o principal fator impulsionador das mudanças climáticas globais atuais e que, a fonte dos GEE é, principalmente, resultado da queima de combustíveis fósseis e das mudanças no uso da terra operacionalizadas pelo homem.

Mesmo sendo tratadas como um fenômeno global, as mudanças climáticas já podem ser sentidas e observadas em escalas regional e local em praticamente todos as partes do mundo (Brown et al., 2012). Destacando o Brasil, o país tem se deparado com uma crescente demanda por estudos sobre as mudanças climáticas e seus efeitos na população, biodiversidade, agropecuária etc. Em resposta a essa demanda, o Primeiro Relatório de Avaliação Nacional sobre Mudanças Climáticas (PBMC, 2014a, b) fez predições de aumento da temperatura do ar e maior frequência de eventos climáticos extremos para todo território nacional, com impactos negativos significativos sobre vários setores (agricultura, biodiversidade, infraestrutura, saúde, educação etc.) e em todos as regiões do país. O Relatório salientou também que uma das regiões do país mais vulnerável do ponto de vista socioeconômico e ambiental, é a região Nordeste.

Para a região Nordeste e sua porção semiárida foi projetado uma tendência de aumento no número de meses com déficit hídrico (aridização), considerando um cenário pessimista (Marengo, 2008 e 2014; Sales et al., 2015; Lacerda et al., 2016 e Marengo et al., 2017). À sua vez, para a Caatinga, que é a cobertura vegetal dominante na Região ou em seu sentido mais amplo chamada de bioma Caatinga, foi projetado também considerando um cenário pessimista (RCP8.5), aumento da temperatura do ar próximo à superfície em $4,0{ }^{\circ} \mathrm{C}$ e redução na precipitação de $0.3 \mathrm{~mm} \mathrm{dia}^{-1}$ até 2100 (Torres et al., 2017).

Essas previsões trazem preocupações ambientais, haja vista o diagnóstico do Quinto Relatório Nacional sobre a Biodiversidade (BRASIL, 2016a) que classificou o bioma Caatinga como o quarto mais devastado do país, com $53,4 \%$ de vegetação nativa remanescente, e por ter sido apontado junto com o bioma Amazônia, como uma das regiões ecológicas do mundo mais vulnerável às mudanças climáticas previstas para este século (Baettig et al., 2007; Santos et al., 2014; Seddon et al., 2016). Ademais, as previsões trazem preocupações socioeconômicas, tendo em conta o bioma Caatinga ser o lugar onde vivem 28,6 milhões de pessoas (Silva et al., 2017). Portanto, o bioma que já enfrenta várias adversidades antrópicas, sentirá de forma mais intensa os efeitos das mudanças climáticas até o final do século, com prejuízos incalculáveis para os ecossistemas e os sistemas humanos e sociais.

As mudanças climáticas estão em curso, continuarão atingindo o bioma e os estudos disponíveis são insuficientes para uma avaliação adequada de suas consequências ambientais e socioeconômicas. Esse fenômeno é mais um enorme desafio a ser enfrentado, sobretudo, pela população residente no bioma, que pode ter suas atividades cotidianas alteradas. Vale destacar que as regiões áridas e semiáridas do mundo, principalmente, aquelas em que existe alto índice de pobreza, sofrerão mais ainda com os efeitos negativos das mudanças climáticas globais no decorrer do século XXI (IPCC, 2014a,b). Então, é oportuno perguntar: Como a população residente no bioma Caatinga percebe as mudanças no clima?

Diante da crise ambiental e climática a percepção do meio ambiente pela população torna-se importante, especialmente, para aquelas populações que vivem em áreas vulneráveis, pois a percepção da gravidade da situação pode representar um relevante elemento para a busca de estratégias e alternativas de convivência com o local e, também, de adaptação às mudanças climáticas (Andrade et al., 2014; Pedrini et al., 2016).

A percepção e o saber ambiental podem colaborar sobremaneira para com a formação de sujeitos críticos, reflexivos, participativos e conscientes de seus papéis na sociedade, além da formulação de ações integradas que agrupem os seres humanos em todas as suas dimensões (biológica, psicológica, social e espiritual) e em um convívio pleno com a natureza (Orsi et al., 2015). Daí a necessidade de um processo educativo e ambiental que leve os indivíduos a se perceberem como seres integrantes e corresponsáveis pelo meio ambiente.

A percepção acerca da importância da conservação e uso sustentável da natureza pode ser alcançada por meio da educação oferecida de modo formal ou informal. Logo, a escola torna-se um espaço importante no processo formal, sendo uma de suas funções formar cidadão para o 
futuro, munindo-o de conhecimento e dando-o suporte para construir uma sociedade melhor para as gerações futuras. Para alcançar esse objetivo, é necessário que a escola trabalhe a temática ambiental de maneira efetiva, desenvolvendo atividades pedagógicas voltadas à conservação da natureza na qual está inserida, direcionando os alunos para a cidadania ativa, considerando o seu sentido de pertencimento e corresponsabilidade que, por meio da ação coletiva e organizada, busca a superação dos problemas ambientais.

Para tanto, é imprescindível que todos os sujeitos da comunidade escolar estejam engajados nesse processo e se percebam como responsáveis pelo meio ambiente. Nesse âmbito, os professores assumem um papel de destaque, uma vez que eles podem promover a conscientização por meio de atividades que estimulem os estudantes a questionar, pesquisar e entender que as mudanças climáticas são um problema cada vez mais presente e que pode atingir a todos, assim contribuindo para a formação do senso crítico e da ação educativa na proteção e no cuidado com o meio ambiente.

Nesse contexto é essencial conhecer os valores que os professores atribuem ao meio ambiente, a forma como percebem as mudanças no clima e as consequências no meio em que estão inseridos. Igualmente, não pode faltar uma verificação se esses profissionais estão preparados para mediar essas discussões, em especial aqueles que atuam nas disciplinas de Ciências e Geografia. Portanto, essa pesquisa teve por objetivo avaliar o nível de percepção dos professores da rede pública do município de General Sampaio (CE), quanto às mudanças climáticas correntes no bioma Caatinga e seus impactos em diferentes setores da sociedade local e perspectivas, tendo em vista contribuir para uma gestão do risco climático no bioma.

\section{Material e Métodos}

A partir de Scientific Electronic Library Online (SciELO) e de repositórios das universidades federais e estaduais do país, consultas foram feitas à obras sobre o fenômeno das mudanças climáticas globais, seus efeitos no bioma Caatinga, as projeções para o futuro próximo e distante e, finalmente, sobre a importância da educação formal e da percepção ambiental no contexto das mudanças climáticas. Na sequência, uma pesquisa de campo foi realizada no período de agosto a novembro de 2019 .

A pesquisa de campo ocorreu nas escolas da rede pública do município de General Sampaio - CE, por meio de entrevistas com professores e utilizando-se de questionários, onde buscou-se analisar a percepção desses professores em relação aos impactos das mudanças climáticas no bioma Caatinga. Nas entrevistas foram consideradas as orientações de Whyte (1977) para pesquisas no âmbito da percepção ambiental e que considera três procedimentos: observando, perguntando e ouvindo.

Além desses pontos considerou-se também as sugestões de Fogaça e Limberger (2014), quando afirmaram que "Nos estudos de percepção ambiental e climática, deve-se levar em consideração o ambiente no qual o participante vive, sua rotina, os estímulos que lhe são causados e como o ambiente [...] pode proporcionar novas experiências e aguçar o saber". Portanto, as questões levantadas durante as entrevistas estavam diretamente ligadas a realidade e ao contexto socioambiental dos participantes.

\subsection{Lócus e sujeitos da pesquisa}

O município escolhido foi General Sampaio que está localizado no estado do Ceará, na microrregião do Médio Curu, a uma distância de $123 \mathrm{~km}$ da capital cearense, Fortaleza. Possui uma extensão territorial de $205,810 \mathrm{~km}^{2} \mathrm{e}$ forma fronteira com os municípios de Apuiarés, Tejuçuoca, Paramoti e Canindé. A população no último censo foi de 6.218 pessoas (IBGE, 2010).

O município encontra-se em uma área de depressão sertaneja e está submetido ao clima semiárido - BSh (Alvares et al., 2014). As temperaturas médias anuais variam entre $26^{\circ} \mathrm{C}$ e $28^{\circ} \mathrm{C}$ e a precipitação oscila em termos médios de 300 a $1.000 \mathrm{~mm}$ anuais (Reddy, 1983). Há um período seco que se estende de 7 a 10 meses sem chuva (Prado, 2003) e a vegetação dominante é a Caatinga.

No âmbito da educação, o município General Sampaio que tem o certificado "Selo UNICEF", conta com um total de 180 professores em exercício, que atendem um número de 1.878 alunos matriculados no ano de 2018 . Possui nove escolas municipais de Educação Infantil e Ensino Fundamental (General Sampaio, 2018). Dentre essas escolas seis foram lócus da pesquisa, sendo elas: EMEF José Bezerra Filho (Sede), EMEIF Manoel Justino Monteiro (Comunidade Lagoa do Meio), EMEF Messias Delfino Alves (Vila São João), EMEIF Raimundo Lessa dos Santos (Localidade de Pedras Pretas), EMEIF Santa Lúcia (Localidade do Pinda) e EMEIF Pascoal de Almeida Sousa (Localidade de Cangati). Das seis escolas, cinco estão localizadas na zona rural do município.

Na zona rural a população trabalha, principalmente, com o cultivo de milho, feijão, mandioca e frutíferas e com a criação de bovinos, caprinos, suínos, aves e peixes (tilápia) (General Sampaio, 2018). Logo, a população dessas comunidades e os estudantes atendidos por essas escolas dependem dos recursos naturais e das condições climáticas para desenvolverem suas atividades de subsistência.

A pesquisa de campo contou com a participação de 10 professores lotados nas escolas supracitadas, especificamente, professores das disciplinas de Geografia e Ciências da Natureza de turmas do $6^{\circ}$ ao $9^{\circ}$ Ano do Ensino Fundamental. 


\subsection{Análise dos dados}

Na pesquisa de campo, as respostas obtidas por meio das entrevistas com os professores, foram analisadas qualitativamente com auxílio do software IRAMUTEQ (Interface de R pour les Analyses Multidimensionnelles de Textes et de Questionnaires ou Interface de R para Análises Multidimensionais de Textos e Questionários), que permite diferentes processamentos e análises de textos, entrevistas e questionários (Lahlou, 2012). O IRAMUTEQ não é especificamente um método de análise de dados, mas uma ferramenta para processá-los. O software não encerra a análise, pois a riqueza do material dependerá das reflexões e da interpretação dos pesquisadores (Lahlou, 2012). Portanto, o IRAMUTEQ se caracteriza como parte de um processo informatizado para análises textuais que busca entender a estrutura e organização do discurso, após processamento de um corpus de texto.

Dentre os recursos disponíveis pelo software foram utilizados aqueles que dão suporte à análise de dados qualitativos como: 1) as estatísticas textuais clássicas, destacando-se a frequência das palavras no corpus e dentro das classes; 2) a classificação hierárquica descendente (CHD), que permitiu a classificação lexical (agrupamento por meio do sentido das palavras) na qual os segmentos de texto e seus vocabulários são correlacionados, assim formando um esquema hierárquico de classes de palavras que, a partir delas foi possível inferir sobre o conteúdo do corpus, nomear cada classe e compreender as ideias colocadas pelos entrevistados; e 3) a nuvem de palavras como um recurso visual que apresenta as palavras mais evocadas nos discursos dos participantes.

Para chegar ao corpus e seguir com a análise, foi necessário a realização das seguintes etapas: 1) transcrição e digitação das entrevistas no Word, formando um documento para cada participante, gerando um total de 10 arquivos de textos, que passou por uma leitura inicial para ter-se uma visão geral das ideias colocadas pelos entrevistados; 2) os 10 arquivos de textos foram dispostos em um único arquivo constituído de nove páginas, passando por uma leitura mais rigorosa que possibilitou os recortes das mensagens e a classificação dos elementos mais relevantes; e 3) esses recortes foram agrupados em um outro documento que compôs o corpus da pesquisa, formado por sete páginas e meia.

Antes do processamento no IRAMUTEQ, o corpus precisou passar por outra edição, sendo transformado em um arquivo TXT, editado no Bloco de Notas do Windows e com formatação mínima - sem negrito, itálico e sublinhado, estruturado como uma sequência de linhas e sem parágrafos, constituindo 10 textos dentro do mesmo arquivo (Fig. 1).

Nessa edição foi colocada a identificação dos entrevistados, idade e sexo (01-Feminino e 02-Masculino), destacada, por exemplo, com a nomenclatura:

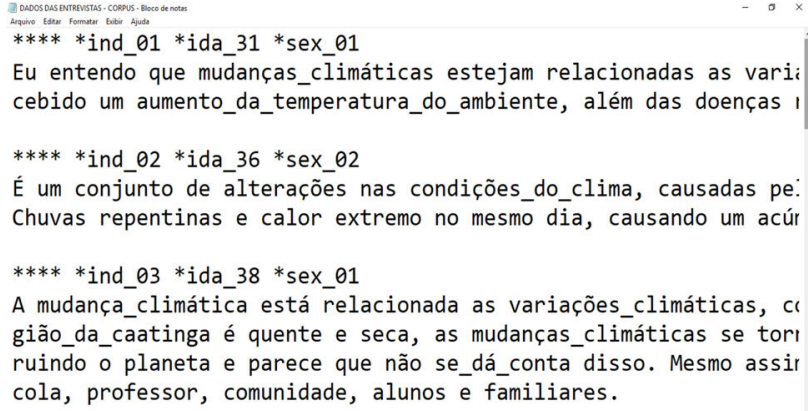

Figura 1 - Recorte do Corpus de texto em TXT para processamento no IRAMUTEQ.

*****ind_01*ida_31*sex_01. E, abaixo de cada uma dessas identificações foi editada, de maneira sequencial, todas as respostas dos respectivos entrevistados. Além disso, algumas palavras e expressões tiveram que ser ligadas com underline “", para não apresentarem sentidos divergentes com as ideias colocadas pelos sujeitos participantes das entrevistas ao ser processado o corpus no IRAMUTEQ.

No software, o corpus transformou-se em segmentos de textos (ST) que são fragmentos (recortes) que, na maioria das vezes, podem ser frases ou parágrafos com até três linhas, dimensionadas pelo próprio software. Do processamento surgiram os resultados de saída (output), sendo utilizados na pesquisa: as estatísticas textuais clássicas; a classificação hierárquica descendente (CHD); e a nuvem de palavras.

Posteriormente, esses resultados foram analisados pelos pesquisadores, passando por interpretações e inferências. Por esse processo indutivo, procurou-se não apenas compreender o sentido da fala dos entrevistados, mais também, os significados que eles atribuíam ao meio e o grau de percepção em relação aos impactos das mudanças climáticas no bioma Caatinga.

\section{Resultados e Discussão}

O corpus processado no IRAMUTEQ foi transformado e separado em 124 segmentos de texto (ST) com aproveitamento de 109 STs $(87,90 \%)$, de onde emergiram 4.290 ocorrências (palavras, formas ou vocábulos). Esses 109 STs aproveitados foram distribuídos em cinco classes, organizadas hierarquicamente como segue: Classe $1 \mathrm{com}$ 26 STs (23,85 \%); Classe 2 com 18 STs (16,51\%); Classe 3 com 18 STs (16,51\%); Classe 4 com 26 STs (23,85\%); e Classe 5 com 21 STs $(19,27 \%)$. Desse modo, foi possível observar que as Classes 1 e 4 apresentaram maior percentual, sugerindo que as palavras que a compõem, foram mais citadas pelos participantes. Além disso, delas emergiram as outras classes (Fig. 2). 
O corpus processado gerou também uma nuvem de palavras que revelou, visualmente, os vocábulos mais evocados pelos participantes das entrevistas (Fig. 3).

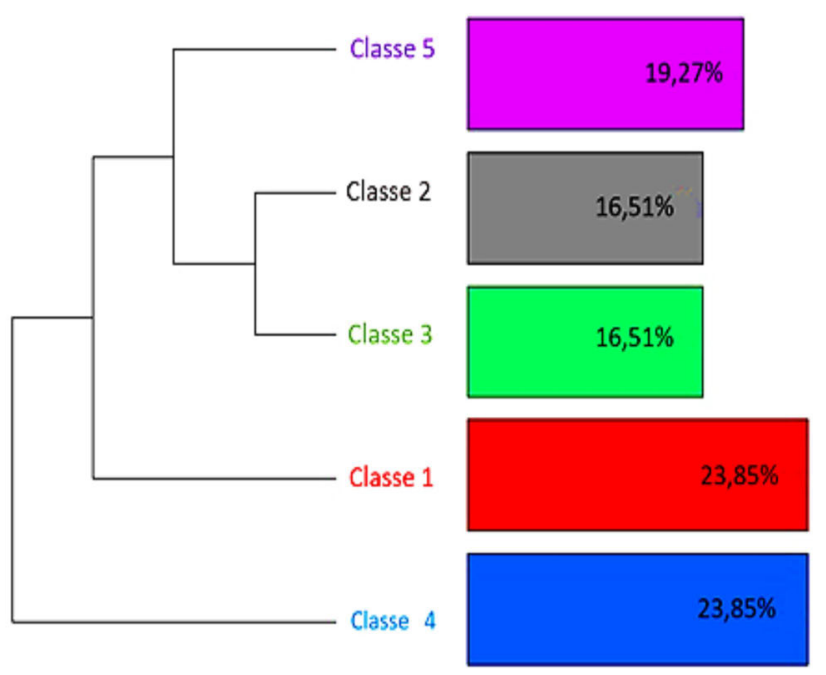

Figura 2 - Dendograma da classificação hierárquica descendente.

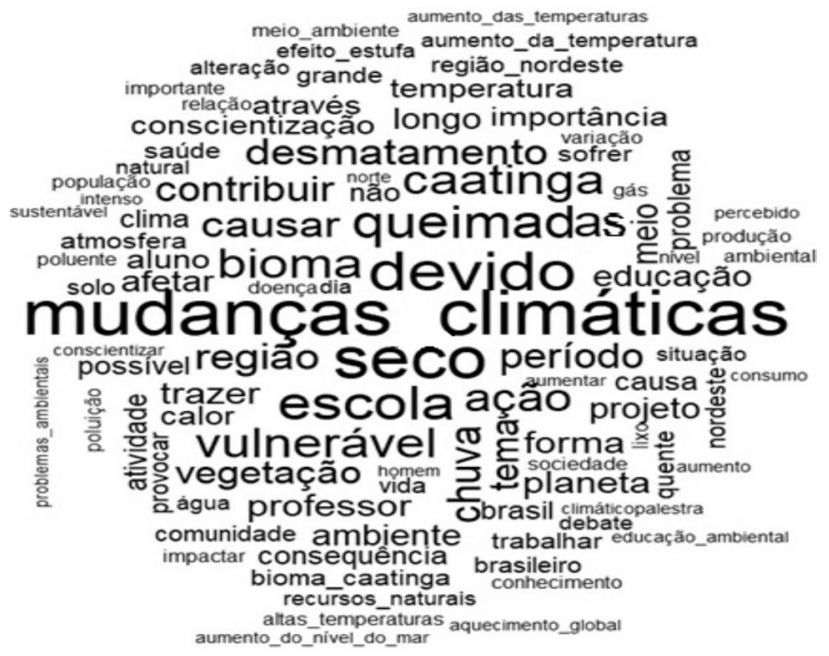

Figura 3 - Nuvem de palavras.
Desse processamento as palavras mais presentes nos discursos dos entrevistados foram: "Mudança climática" ( $\mathrm{f}=28)$; "Seco" ( $\mathrm{f}=25)$; "Bioma" ( $\mathrm{f}=17)$; "Queimadas" $(\mathrm{f}=19)$; "Escola" ( $\mathrm{f}=20)$; "Vulnerável" $(\mathrm{f}=17)$; "Desmatamento" ( $f=15)$; "Caatinga" ( $f=17)$; "Vegetação" $(f=13)$; "Causar" ( $f=15)$; e "Contribuir" ( $f=14)$.

Além disso, produziu-se também dados de estatísticas textuais, que mostraram a frequência (f) das palavras nas falas dos entrevistados dentro das cinco classes e o nível de significância $\left(\mathrm{x}^{2}\right)$ da associação dessas palavras com a classe a qual pertence (Fig. 4).

Com os resultados, foi possível nomear as cinco classes geradas no IRAMUTEQ, interpretar e realizar inferências para cada uma das classes, verificar as relações existentes entre elas, bem como reconhecer nas falas dos entrevistados as suas percepções em relação ao meio ambiente, aos problemas ambientais locais, as mudanças climáticas e seus impactos no bioma Caatinga e, por fim, o papel do professor no contexto das mudanças climáticas.

Com isso, a Classe 5, a primeira da Classificação Hierárquica Descendente (Fig. 2) e nomeada com o título "As consequências das ações antrópicas no meio ambiente", apontou os principais problemas ambientais percebidos e identificados pelos participantes das entrevistas, problemas que foram gerados por ações humanas no meio ambiente. Conforme alguns entrevistados: "o homem está destruindo o planeta e parece que não se dá conta disso" (Ind. 03); "há um aumento significativo do desmatamento e das queimadas, contribuindo para um ar insalubre e tóxico" (Ind. 04); "a poluição e os despejos de esgotos em rios afetam os recursos naturais e a qualidade de vida" (Ind. 02).

Dentre os diversos problemas ambientais gerados pelas atividades humanas, as queimadas e o desmatamento apareceram com maior frequência nas falas dos entrevistados. Possivelmente, isso se deve ao fato desses problemas estarem fortemente presentes na realidade do Brasil, especialmente, na região Nordeste e no estado do Ceará. Estudos sobre a problemática ambiental têm apontado as ações humanas na natureza, como "fator determi-

\begin{tabular}{|l|c|c}
\hline \multicolumn{3}{|c|}{ CLASSE 1 } \\
\hline Palavra & $f$ & $\mathbf{x}^{\mathbf{2}}$ \\
& $f$ & \\
\hline Bioma & 13 & 38,08 \\
\hline Caatinga & 15 & 37,78 \\
\hline Vegetação & 9 & 31,32 \\
\hline Seco & 20 & 17,62 \\
\hline Folhagem & 4 & 13,26 \\
\hline Sofrer & 8 & 12,43 \\
\hline Semiárido & 3 & 9,85 \\
\hline Nordeste & 6 & 6,41 \\
\hline Chuva & 12 & 5,08 \\
\hline População & 5 & 3,77 \\
\hline
\end{tabular}

\begin{tabular}{|l|c|c}
\hline \multicolumn{3}{|c}{ CLASSE 2 } \\
\hline Palavra & $f$ & $\mathbf{x}^{\mathbf{2}}$ \\
& & \\
\hline Planeta & 11 & 19,71 \\
\hline Clima & 9 & 66,67 \\
\hline Mudança & 7 & 16,36 \\
\hline Provocado & 3 & 15,6 \\
\hline Atmosfera & 8 & 13,24 \\
\hline Temperatura & 9 & 10,85 \\
\hdashline Poluente & 4 & 10,85 \\
\hline Global & 4 & 10,30 \\
\hline Causar & 14 & 8,09 \\
\hline Afetar & 11 & 7,43 \\
\hline
\end{tabular}

\begin{tabular}{|l|c|c|}
\hline \multicolumn{3}{|c}{ CLASSE 3 } \\
\hline Palavra & $f$ & $\mathbf{x}^{\mathbf{2}}$ \\
& & \\
\hline Aquecimento & 20 & 20,99 \\
\hline Ef. Estufa & 6 & 20,59 \\
\hline Acelerado & 3 & 15,60 \\
\hline Região & 13 & 14,92 \\
\hline Vulnerável & 17 & 13,63 \\
\hline Aumento & 6 & 11,58 \\
\hline Ar & 3 & 5,63 \\
\hline Gases & 3 & 5,63 \\
\hdashline Afetado & 3 & 5,63 \\
\hline Temperatura & 9 & 5,55 \\
\hline
\end{tabular}

\begin{tabular}{|l|c|c}
\hline \multicolumn{3}{|c}{ CLASSE 4 } \\
\hline Palavra & $f$ & $\mathbf{x}^{2}$ \\
& & \\
\hline Escola & 12 & 43,05 \\
\hline Tema & 12 & 43,05 \\
\hline Professor & 12 & 43,05 \\
\hline Aluno & 10 & 35,15 \\
\hline Trabalhar & 6 & 20,27 \\
\hline Ação & 10 & 19,11 \\
\hline Conscientizar & 5 & 16,73 \\
\hline Aula & 5 & 16,73 \\
\hline Debate & 5 & 16,73 \\
\hline Comunidade & 3 & 9,85 \\
\hline
\end{tabular}

\begin{tabular}{|l|c|c|}
\hline \multicolumn{3}{|c|}{ CLASSE 5 } \\
\hline \multirow{2}{*}{ Palavra } & $f$ & $\mathbf{x}^{\mathbf{2}}$ \\
& $f$ & \\
\hline Perceber & 10 & 35,42 \\
\hline Poluição & 44 & 17,40 \\
\hline Dia & 6 & 16,76 \\
\hline Ar & 6 & 16,76 \\
\hline Lixo & 5 & 12,43 \\
\hline Calor & 10 & 60,00 \\
\hline Saúde & 8 & 10,38 \\
\hline Quente & 8 & 10,38 \\
\hline Problema & 8 & 9,17 \\
\hline Homem & 4 & 8,29 \\
\hline
\end{tabular}

Figura 4 - Estatística textual: frequência e nível de significância das palavras. 
nante de mudanças na dinâmica dos ecossistemas, com consequências negativas sobre a biodiversidade, as condições climáticas, nos seres humanos e no seu modo de vida" (Santos et al., 2017).

A Classe 5 ainda apresentou uma correlação com a Classe 2, intitulada "Percepção sobre as mudanças climáticas globais", em que os entrevistados afirmaram que as atividades humanas são as principais causas das mudanças climáticas, provocadas pelo "consumismo desenfreado que por sua vez influencia na produção a qualquer custo" (Ind. 01), por "políticas globalizadas e até mesmo por ignorância às leis da natureza" (Ind. 09).

Para a grande maioria dos entrevistados, as mudanças climáticas estão relacionadas as alterações nos padrões do clima causadas pelas atividades humanas tais como queimadas, desmatamento, industrialização, poluição, consumismo, que elevam os níveis de concentração de gases de efeito estufa (GEE) na atmosfera, provocando o aquecimento do planeta.

Alguns entrevistados afirmaram ainda que percebem as variações no clima através da "radiação solar que parece muito mais forte" (Ind. 07), dos "dias cada vez mais quentes e secos" (Ind. 05), das "sensações térmicas inconstantes, do calor intenso e das secas extremas" (Ind. 10), assim como de "chuvas fora de época, ventos fortes e maior ocorrência de desastres ambientais" (Ind. 02).

De acordo com o Painel Brasileiro de Mudanças Climáticas (PBMC, 2014 a), a principal causa das mudanças climáticas está relacionada a emissão de GEE, em especial o dióxido de carbono, gerada a partir de atividades antrópicas, que estão alterando os sistemas naturais e humanos. As consequências já podem ser verificadas nas mudanças de temperatura, nos índices pluviométricos, perda de biodiversidade, ocorrência de extremos climáticos etc.

A Classe 3, nomeada "Impactos das mudanças climáticas", está relacionada às consequências das mudanças climáticas verificadas e colocadas pelos entrevistados, conforme citado: "[...] podem impactar na minha vida, principalmente, pelas altas temperaturas e a escassez de água potável" (Ind. 06); "Pode afetar a saúde devido ao sol forte, causando problemas de pele e devido à seca e as chuvas em períodos irregulares, que causam problemas respiratórios" (Ind. 10); "Pois interferem na qualidade de vida, na saúde, no convívio, na mobilidade, no lazer, na prática de esportes e outras atividades" (Ind. 08).

De acordo com os entrevistados, o clima local interfere diretamente não só nos ambientes naturais, mais ainda nos modos de vida das pessoas, tendo em vista que os costumes, alimentação, saúde, economia, transporte, atividades de lazer, dentre outras, dependem também das condições ambientais e climáticas. Portanto, ocorrendo alterações nos padrões climáticas, consequentemente, isso vai gerar problemas em vários setores da sociedade. Portanto, "O impacto da mudança do clima é tratado como um dos fatores que contribuem para o aumento dos riscos de desastres naturais", que podem resultar na ocorrência de eventos extremos (BRASIL, 2016b). A previsão é que "[...] essas mudanças afetem os sistemas naturais, humanos, de infraestrutura e produtivo do país" (BRASIL, 2016b). Por isso, têm-se mostrado um dos maiores desafios atuais da humanidade, tornando imprescindível o desenvolvimento de estratégias de adaptação.

Na sequência, a Classe 1, nomeada "Percepção sobre o bioma Caatinga", foi destacada a forma como os entrevistados veem e caracterizam a Caatinga, além de manifestar sobre a vulnerabilidade do bioma em relação às mudanças climáticas. Aqui, em suma, afirmaram que a Caatinga está localizada na região Nordeste do Brasil, sendo um ecossistema próprio do clima semiárido, com vegetação espinhosa e pouca folhagem. Foi comentado ainda que "[...] para muitas pessoas a Caatinga é um bioma seco e sem vida" (Ind. 05) e que "[...] sofre com o desmatamento, as queimadas e as secas" (Ind. 10).

Muitos dos entrevistados revelaram desconhecer particularidades do bioma Caatinga, como os elementos naturais de sobrevivência das populações residentes e, principalmente, a diversidade biológica que marca sua heterogeneidade. A Caatinga é um bioma exclusivamente brasileiro, compreendendo grande parte do semiárido nordestino e que apresenta uma elevada riqueza de espécies animal e vegetal, além de muitas espécies endêmicas (Souza et al., 2015; Gusmão et al., 2016).

Os entrevistados afirmaram ainda que a vulnerabilidade do bioma às mudanças climáticas está "relacionada aos períodos de seca, cada vez maiores" (Ind. 07), que ocorre "devido suas próprias características quente e seco" (Ind. 06) e que vem se agravando, principalmente, "por conta do desmatamento, das queimadas e das secas constantes" (Ind. 10). É possível verificar também que os entrevistados colocaram a estiagem como fator principal que torna o bioma Caatinga mais vulnerável às mudanças climáticas. De fato, várias pesquisas já apontaram nessa direção (Marengo, 2008; Santos et al., 2014; Marengo et al., 2017), mas acrescenta-se a isso os problemas socioeconômicos locais e a desertificação. Além disso, a vulnerabilidade na região em estudo está relacionada ao acréscimo da temperatura do ar, a diminuição da precipitação, as ocorrências de dias mais secos e de mais ondas de calor (Marengo, 2008).

Vale ainda destacar que o bioma abrange também ecossistemas particularmente mais frágeis às alterações do clima, condições potencializadas por características dos solos, exploração inadequada dos recursos naturais e ao superpastoreio. Há também que considerar o nível de pobreza da região Nordeste, que impossibilita às populações locais de ter acesso aos recursos necessários para o enfretamento das alterações climáticas (Araújo e Sousa, 2011).

Diante desses cenários, a educação pode contribuir significativamente no processo de conscientização dos 
agentes sociais, levando informações e conhecimentos para a população sobre assuntos relacionados as questões ambientais e as mudanças do clima, e contribuir com ações sustentáveis e orientações sobre a importância de conservar os recursos naturais.

A última classe, Classe 4 "A Importância da Educação no Contexto das Mudanças Climáticas", os entrevistados relataram sobre o papel da escola e dos professores frente aos impactos das alterações do clima, destacando a relevância da educação para a formação de sujeitos comprometidos com a causa ambiental e, sobretudo, com a emergência climática.

Para os professores: "a escola pode contribuir na formação de sujeitos responsáveis pelo meio ambiente, desenvolvendo projetos que trabalhem a conscientização" (Ind. 01); realizando "debates e leituras sobre o tema, orientando o estudante do seu papel ambiental, criando e implantando projetos de educação ambiental" (Ind. 03); levando para as salas de aula "atividades e informações sobre o bioma Caatinga e sobre as mudanças climáticas" (Ind. 05). Além disso, todas essas ações podem ocorrer "a partir da implantação da educação ambiental no currículo, no plano de ensino e no planejamento das aulas" (Ind. 10). Os entrevistados também reconheceram a importância da educação no processo de conscientização dos indivíduos e da Educação Ambiental como uma prática a ser trabalhada de forma interdisciplinar e efetiva nas escolas e pelos professores.

Portanto, a educação tem um importante papel na formação de cidadãos conscientes e responsáveis pelo meio ambiente e, a educação ambiental surge como uma ferramenta importante nesse processo, podendo estar presente em toda a ação educativa, seja ela informal ou formal (Barboza et al., 2016). No caso do ambiente escolar pode ser desenvolvida como uma prática integrada, contínua e permanente em todos os níveis e modalidades do ensino. Assim, os professores podem trabalhar a questão climática de modo interdisciplinar, realizando a interação de sua disciplina com as questões ambientais, desenvolvendo diferentes metodologias e atividades (Viana, 2006).

\section{Considerações Finais}

As mudanças climáticas vêm afetando o planeta e transformando, paulatinamente, os sistemas naturais e humanos. Embora o fenômeno seja global, seus impactos são sentidos e observados em escala local, principalmente, em regiões vulneráveis onde outros problemas socioambientais já são vivenciados por suas populações.

O bioma Caatinga é considerado uma região ecológica vulnerável no Brasil, enfrentando longos períodos de estiagem, desmatamentos, queimadas, desertificação etc. e onde parte significativa da população ainda carece de elementos básicos como saúde, moradia, alimentação, água potável e educação formal. Acrescenta-se a esse quadro considerando os resultados da pesquisa, que embora os professores da rede pública do município de General Sampaio (CE) tenham formação nas áreas de Ciências da Natureza e Geografia, percebam os problemas ambientais causados pelas ações antrópicas e admitam impactos negativos das mudanças climáticas onde vivem, eles ainda carecem de conhecimento suficiente sobre o bioma Caatinga, às mudanças climáticas globais e seus impactos negativos em escala local. Portanto, é uma situação preocupante, tendo em vista a possibilidade de implementação de medidas adaptativas na população em um futuro próximo.

Nessa perspectiva, fortalecer a educação formal e, consequentemente a informal, pode contribuir para a formação de uma consciência e responsabilidade ambiental. Todavia, para que se alcance a formação supracitada, as escolas da rede pública do município de General Sampaio (CE) devem trabalhar regularmente os temas em aulas, projetos, atividades e ações, levando-se em consideração as características naturais do lugar em que estão inseridas e os problemas socioambientais encontrados na região. Evidentemente, é central que primeiro os professores se capacitem.

Vale ainda destacar que, essa fragilidade no conhecimento a cerca do tema Mudanças Climáticas por parte dos professores do município de General Sampaio - CE, certamente, também se verifique em muitos outros municípios inseridos no bioma Caatinga. Assim, de modo mais abrangente, sugere-se que gestores de todas as esferas governamentais promovam um estímulo geral e coordenado, envolvendo todos os atores protagonistas do âmbito escolar, podendo desenvolver projetos de formação ambiental para professores e alunos, levando em consideração as características locais, os problemas socioambientais encontrados na região, as possíveis formas de uso do bioma e o seu desenvolvimento sustentável.

Essa ação poderá encorajar e impulsionar ideias e atitudes de conservação e sustentabilidade em todo o bioma. Como premissa, a conscientização de todos em se reconhecer como parte do meio ambiente e responsáveis por sua conservação. Isso favoreceria à alfabetização climática e, consequentemente, a resiliência e a adaptação das populações diante da real ameaça das mudanças climáticas.

\section{Referências}

ALVARES, C.A.; STAPE, J.L.; SENTELHAS, P.C.; GONÇALVES, J.L.M.; SPAROVEK, G. Köppen's climate classification map for Brazil. Meteorologische Zeitschrift, v. 22, n. 6, p. 711-728, 2014.

ANDRADE, A.J.P.; SILVA, N.M.; SOUZA, C.R. As percepções sobre as variações e mudanças climáticas e as estratégias de adaptação dos agricultores familiares do Seridó potiguar. Desenvolvimento e Meio ambiente, v. 31, p. 77-96, 2014.

ARAÚJO, C.S.F.; SOUSA, A.N. Estudo do processo de desertificação na Caatinga: Uma proposta de educação ambiental. 
Ciência \& Educação, Campina Grande, v. 17, n. 4, p. 975986, 2011.

BAETTIG, M.B.; WILD, M.; IMBODEN, M.D.A climate change index: where climate change may be more prominent in the 21st century. Geophysical Research Letters, v. 34, n. 01705, p. 1-6, 2007.

BARBOZA, L.A.S.; BRASIL, D.S.B.; CONCEIÇÃO, G.S. Percepção ambiental dos alunos do $6^{\circ}$ e do $9^{\circ}$ anos de uma escola pública municipal de Redenção, Estado do Pará, Brasil. Revista Pan-Amazônica de Saúde, v. 7, n. 4, p. 1120, 2016.

BRASIL. Ministério do Meio Ambiente. $5^{\circ}$ Relatório Nacional para a Convenção Sobre Diversidade Biológica. Brasília: MMA, 2016a. 240 p.

BRASIL. Ministério do Meio Ambiente. Plano Nacional de Adaptação à Mudança do Clima. Brasília: MMA, 2016b. $295 \mathrm{p}$.

BROWN, A.; DAYAL, A.; RIO, C.R.D. From practice to theory: emerging lessons from Asia for building urban climate change resilience. Environment and Urbanization, v. 24, n. 4, p. 531-556. 2012.

FOGAÇA, T.K.; LIMBERGER, L. Percepção ambiental e climática: estudo de caso em colégios públicos do meio urbano e rural de Toledo-PR. Revista do Departamento de Geografia-USP, v. 28, p. 134-156, 2014.

GENERAL SAMPAIO. Portal da Prefeitura Municipal de General Sampaio. Disponível em: http://www.generalsam paio.ce.gov.br/informacoesGeograficas. Acesso em: 02 jul. 2018.

GUSMãO, L.F.P.; QUEIROZ, L.P.; QUIJANO, F.R.B.; JUNCá, F.A.; OLIVEIRA, R.P.; BASEIA, I.G. Caatinga - Diversidade na adversidade do semiárido brasileiro. In: PEIXOTO, A.L.; LUZ, J.R.P.; BRITO, M.A. Conhecendo a Biodiversidade. Brasília: MCTIC, CNPq, PPBio, 2016.

IBGE - Instituto Brasileiro de Geografia e Estatística. Censo 2010. Disponível em: https://ibge.gov.br/brasil/. Acesso em: 02 jul. 2018.

IPCC - Intergovernmental Panel on Climate Change. Climate Change 2014: Impacts, Adaptation, and Vulnerability. Part A: Global and Sectoral Aspects. Contribution of Working Group II to the Fifth Assessment Report of the Intergovernmental Panel on Climate Change. FIELD, C.B.; BARROS, V.R.; DOKKEN, D.J.; MACH, K.J.; MASTRANDREA, M.D. et al. (eds). Cambridge University Press: Cambridge, United Kingdom and New York, 2014a, 1132 p.

IPCC - Intergovernmental Panel on Climate Change. Climate Change 2014: Impacts, Adaptation, and Vulnerability. Part B: Regional Aspects. Contribution of Working Group II to the Fifth Assessment Report of the Intergovernmental Panel on Climate Change. BARROS, V.R.; FIELD, C.B.; DOKKEN, D.J.; MASTRANDREA, M.D.; MACH, K.J. et al. (eds). Cambridge University Press: Cambridge, United Kingdom and New York, 2014b, $688 \mathrm{p}$.

IPCC - Intergovernmental Panel on Climate Change. Climate Change 2013: The Physical Science Basis. Contribution of Working Group I to the Fifth Assessment Report of the Intergovernmental Panel on Climate Change.
STOCKER, T.F.; QIN, D.; PLATTNER, G.K.; TIGNOR, M.; ALLEN, S.K. et al. (eds). Cambridge University Press: Cambridge, United Kingdom and New York, 2013, 1535 p.

IRAMUTEQ - Interface de $\mathbf{R}$ pour les Analyses Multidimensionnelles de Textes et de Questionnaires. Disponível em: https://sourceforge.net/projects/iramuteq/. Acesso em: 03 jul. 2019.

LACERDA, F.F.; NOBRE, P.; SOBRAL, M.C.M.; LOPES, G.M.B.; ASSAD, E.D. Tendência do clima do semiárido frente as perspectivas das mudanças climáticas globais; o caso de Araripina, Pernambuco. Revista do Departamento de Geografia USP, v. 31, p. 132-141, 2016.

LAHLOU, S. Text Mining Methods: An Answer to Chartier and Meunier. 2012. Disponível em: http://www.psych.lse. ac.uk/psr/. Acesso em: 30 set. 2019.

MARENGO, J.A. Vulnerabilidade, impactos e adaptação à mudança do clima no semiárido do Brasil. Parcerias Estratégicas, v. 13, n. 27, p. 149-175, 2008.

MARENGO, J.A. O futuro clima do Brasil. Revista USP, n. 103, p. 25-32, 2014.

MARENGO, J.A.; TORRES, R.R.; ALVES, L.M. Drought in Northeast Brazil: past, present and future. Theoretical and Applied Climatology, v. 129, n. 3-4, p. 1189-1200, 2017.

ORSI, R.F.M.; WEILER, J.M.A.; CARLETTO, D.L.; VOLOSZIN, M. Percepção ambiental: Uma experiência de ressignificação dos sentidos. Revista Eletrônica do Mestrado em Educação Ambiental da Universidade Federal do Rio Grande-FURG, v. 32, n. 1, p. 20-38, 2015.

PBMC - Painel Brasileiro de Mudanças Climáticas. Base Científica das Mudanças Climáticas. Contribuição do Grupo de Trabalho 1 do Painel Brasileiro de Mudanças Climáticas ao Primeiro Relatório de Avaliação Nacional sobre Mudanças Climáticas. AMBRIZZI, T.; ARAUJO, M. (eds). Coppe/Universidade Federal do Rio de Janeiro: Rio de janeiro, 2014a, $464 \mathrm{p}$.

PBMC - Painel Brasileiro de Mudanças Climáticas. Impactos, Vulnerabilidades e Adaptação às Mudanças Climáticas. Contribuição do Grupo de Trabalho 2 do Painel Brasileiro de Mudanças Climáticas ao Primeiro Relatório da Avaliação Nacional sobre Mudanças Climáticas. ASSAD, E.D.; MAGALHÃES A.R. (eds). Coppe/Universidade Federal do Rio de Janeiro: Rio de Janeiro, 2014b, $414 \mathrm{p}$.

PEDRINI, A.G.; BROTTO, D.S.; SANTOS, T.V.; LIMA, T.; NUNES, R.M. Percepção ambiental sobre as mudanças climáticas globais numa praça pública na cidade do Rio de Janeiro (RJ, Brasil). Revista Ciência e Educação, v. 22, n. 4, p. 1027-1044, 2016.

PRADO, D. As Caatingas da América do Sul. In: LEAL, I.R.; TABARELLI, M.; SILVA, J.M.C. (eds). Ecologia e conservação da Caatinga. Recife: Editora Universitária da UFPE, 2003, pp. 3-73.

REDDY, S.J. Climatic classification: the semi-arid tropics and its environment a review. Embrapa Semiárido Artigo em periódico indexado ALICE, v. 18, n. 8, p. 823-847, 1983.

SALES, D.C.; COSTA, A.A.; SILVA, E.M.; VASCONCELOS JÚNIOR, F.C.; CAVALCANTE, A.M.B. et al. Projeções de mudanças na precipitação e temperatura no nordeste brasileiro utilizando a técnica de downscaling dinâmico. 
Revista Brasileira de Meteorologia, v. 30, n. 4, p. 435456, 2015.

SANTOS, M.G.; OLIVEIRA, M.T.; FIGUEIREDO, K.V.; FALCÃO, H.M.; ARRUDA, E.C. et al. Caatinga, the Brazilian dry tropical forest: can it tolerate climate changes? Theoretical and Experimental Plant Physiology, v. 26, n. 1, p. 83-99, 2014.

SANTOS, P.J.A.; SILVA, M.M.P.; COUTO, M.G.; BORGES, V.G. Relação entre percepção ambiental de docentes e discentes do ensino fundamental II de uma escola pública do semiárido paraibano com características do bioma Caatinga. Revista Eletrônica do Mestrado em Educação Ambiental da Universidade Federal do Rio GrandeFURG, v. 30, n. 1, p. 38-53, 2017.

SEDDON, A.W.; MACIAS-FAURIA, M.; LONG, P.R.; BENZ, D.; WILLIS, K.J. Sensitivity of global terrestrial ecosystems to climate variability. Nature, v. 531, n. 7593, p. 229$232,2016$.
SILVA, J.M.C.; LEAL, I.R.; TABARELLI, M. (eds.). Caatinga: The Largest Tropical Dry Forest Region in South America. new York: Springer, 482 p., 2017

SOUZA, I.B.; ARTIGAS, R.C.; LIMA, E.R.V. Caatinga e desertificação. Mercator, v. 14, n. 1, p. 131-150, 2015.

TORRES, R.R.; LAPOLA, D.M.; GAMARRA, N.L.R. Future Climate Change in the Caatinga. In: SILVA, J.M.C.; LEAL, I.R.; TABARELLI, M. (Eds.). Caatinga: The Largest Tropical Dry Forest Region in South America. New York, Springer, p. 383-410, 2017.

VIANA, P.A.M.O. A inclusão do tema meio ambiente nos currículos escolares. Revista Eletrônica do Mestrado em Educação Ambiental, v. 16, p. 1-17, 2006.

WHYTE, A.V.T. Guidelines for Fields Studies in Environmental Perception. Paris: Unesco, 1977.

License information: This is an open-access article distributed under the terms of the Creative Commons Attribution License (type CC-BY), which permits unrestricted use, distribution and reproduction in any medium, provided the original article is properly cited. 